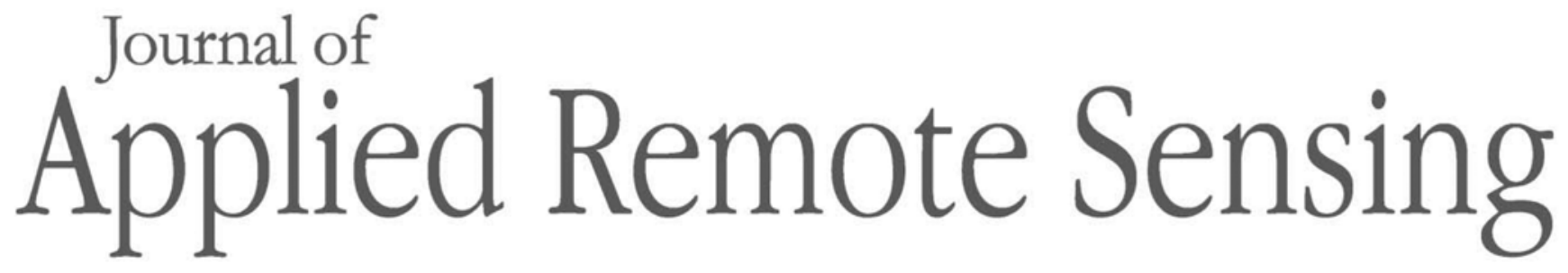

RemoteSensing.SPIEDigitalLibrary.org

\title{
Noise reduction and destriping using local spatial statistics and quadratic regression from Hyperion images
}

Mahendra K. Pal

Alok Porwal

Thorkild M. Rasmussen 


\title{
Noise reduction and destriping using local spatial statistics and quadratic regression from Hyperion images
}

\author{
Mahendra K. Pal, ${ }^{\mathrm{a}, \mathrm{b}, *}$ Alok Porwal, ${ }^{\mathrm{b}}$ and Thorkild M. Rasmussen ${ }^{\mathrm{a}}$ \\ ${ }^{a}$ Luleå University of Technology, Division of Earth Science and Environmental Engineering, \\ Department of Civil, Environmental and Natural Resources Engineering, Luleå, Sweden \\ b Indian Institute of Technology Bombay, Center of Studies in Resources Engineering, \\ Mumbai, Maharashtra, India
}

\begin{abstract}
Hyperion images from Earth Observing-1 (EO-1) are being used in natural resources assessment and management. The evaluation and verification of Hyperion images for the above applications are validating the EO-1 mission. However, the presence of random and striping noises in Hyperion images affect the accuracy of the results. Therefore, reduction of random noise and stripes from Hyperion images becomes indispensable for the evaluation of the results in natural resources assessment and in optimum use of the data. Thus, a collective approach for correcting pixels with no-data values and removing random noise and stripes from Hyperion radiance images is developed. In the developed method, first, no-data valued pixels are identified and corrected using a local median filter. Minimum noise fraction transformation is then used to reduce random noise from noise-dominated bands. Further, spatial statistical techniques are used to reduce random noise from the rest of the bands. Finally, a local quadratic regression by a least squares method is used to correct bad columns and global stripes, and a local-spatial-statisticsbased algorithm is used to detect and correct local stripes. The effectiveness and efficiency of the algorithm is demonstrated by application to two Hyperion images: one from the Udaipur area, western India, and another from the Luleå area, northern Sweden. The results show that the algorithm reduces random and striping noise without introducing unwanted effects and alterations in the original normal data values in the images. (C) The Authors. Published by SPIE under a Creative Commons Attribution 4.0 Unported License. Distribution or reproduction of this work in whole or in part requires full attribution of the original publication, including its DOI. [DOI: 10.1117/1.JRS.14 .016515]
\end{abstract}

Keywords: remote sensing; hyperspectral; Hyperion; minimum noise fraction; least-square regression; spatial statistics; spectral noise.

Paper 190874 received Nov. 7, 2019; accepted for publication Mar. 3, 2020; published online Mar. 19, 2020.

\section{Introduction}

Hyperion, a hyperspectral sensor, is on board the National Aeronautics and Space Administration's Earth Observing-1 (NASA-EO-1) satellite. Hyperion sensor data are the only globally available spaceborne hyperspectral system, which provides hyperspectral Earth exploration data for better-quality characterization of Earth's surface, particularly for the determination of the composition of surface material for geological applications ${ }^{1}$ and natural resources assessments and managements. The distributed Hyperion datasets are radiometrically corrected and orthorectified as level 1A datasets. Level 1A (L1R) datasets are generated from level 0 datasets through the following preprocessing steps: echo correction, smear correction, background removal, bad pixel repair, radiometric correction, and finally checking the image quality. ${ }^{2,3}$ The above corrections, in principle, are expected to recover untrustworthy/noisy pixels, though even a cursory visual inspection of a typical Hyperion L1R image shows this is not the case. Salt-andpepper/random noise and vertical stripes (spatially nonuniformity) appear in most of the bands in Hyperion images with varying amounts. In particular, bands close to the water vapor, oxygen,

*Address all correspondence to Mahendra K. Pal, Email: mahendra.pal@1tu.se 
Pal, Porwal, and Rasmussen: Noise reduction and destriping using local spatial statistics...

and carbon dioxide absorption wavelengths are dominated by random noise as well as stripes. ${ }^{4,5}$ Accurate detection and correction of random noise and vertical stripes are essential, because pixels could possibly exhibit spikes and spurious absorption features in spectra, which may mislead spectral analyses for qualitative and quantitative interpretations.

\section{Nature and Sources of Noise in Hyperspectral Images}

Salt-and-pepper noise and striping are the most frequently encountered noises in Hyperion images. Predominant sources of salt-and-pepper noise and vertical stripes in Hyperion images are attributable to degradation and/or failure of detectors over time, malfunctioning of the detector, separate detector's inaccurate cocalibration on the focal plane array, and/or improper calibration $^{2,6,7}$ and fluctuation in illumination conditions and atmospheric perturbations such as physical disturbances. ${ }^{2,8}$

\subsection{Salt-and-Pepper Noise}

Hyperion images are composed of many narrow adjoining bands. The narrowness of the bands decreases the signal strength and increases noise components. These band-specific noisy pixels due to random and band-correlated noise can cause negative or positive spikes in the spectra. Random noise is manifested as a salt-and-pepper appearance characterized by randomly distributed abnormal bright or dark pixels. Dark pixels are the result of very low or nil sensor response, whereas bright pixels have abnormally high sensor response. ${ }^{9}$ The salt-and-pepper type of noise is mostly dominant in water absorption and nearby bands \{e.g., Fig. 1(a) [band 121 (4), band 224 (6)]\}. Random noise reduction from Hyperion images is critical before destriping.

\subsection{Vertical Stripes}

Vertical stripes are attributed to columns that largely comprise abnormal dark pixels and/or bright pixels. They appear with varying shades of gray as vertical stripes on hyperspectral (Hyperion) images.

Four types of stripes are recognized with different compositions with digital numbers (DN) in Hyperion images: (1) continuous with atypical values \{e.g., Fig. 1(a) [band 201 (5)], Fig. 1(b)
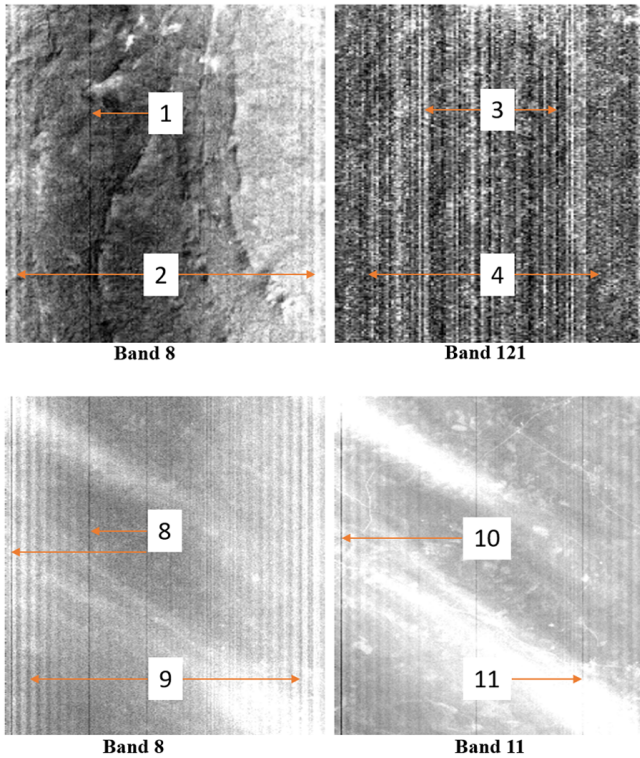

(b)
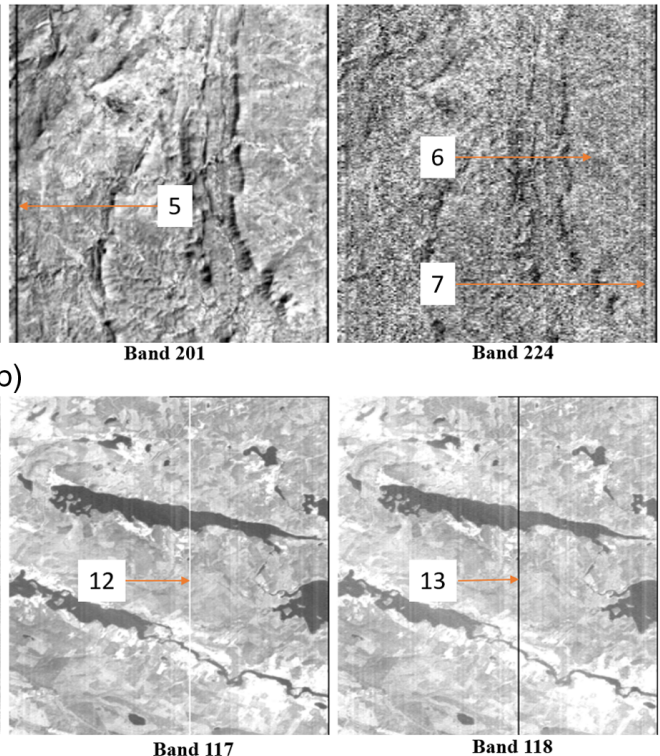

Fig. 1 An example for demonstration of random noise and various types of vertical stripes occurring in Hyperion images: (a) From the Udaipur scene and (b) from the Luleå scene. 
[band 117 (12), band 118 (13)] \}; (2) continuous with constant values \{Fig. 1(a) [band 8 (1), band 121 (3)], Fig. 1(b) [band 8 (8), band 11 (10)]\}; (3) intermittent with atypical values \{Fig. 1(a) [band 224 (7)], Fig. 1(b) [band 11 (11)]\}, and (4) intermittent with lower values \{Fig. 1(a) [band 8 (2)], Fig. 1(b) [band 8 (9)]\}. The first and third types of stripes can be easily identified since they contain abnormal pixels by the extremity of their DN values. The second type of stripes is also easily detected, because they appear across an entire column with abnormal pixels. But the fourth type of stripe is more difficult to detect because they contain abnormal pixels values very close to the normal pixels' values. These appear in different lengths and are not visually evident. These stripes can be confused with columns comprised of normal pixels and real vertical ground features. ${ }^{2}$

Bouali also classified noise and stripes present in remote sensing images. According to Bouali, ${ }^{10}$ remotely sensed images contain two types of stripes. First is the periodic type stripe. This type of stripe is generated by improper offset and relative gain radiometric calibration of the individual detectors in the acquisition system \{Fig. 1(a) [band 8 (2)], Fig. 1(b) [band 8 (9)]\}. The occurrence of periodic stripes may also be due to the fine variations in the slit width in the detector's optical system. An additional reason may be due to input and output transfer function's deviations remaining constant with time with neighboring elements of the detector's matrix. Second is the random type stripe. This type of stripe is triggered by random fluctuations and thermal effects in the sensor responses \{Fig. 1(a) [band 8 (1), band 121 (3)], Fig. 1(b) [band 8 (8), band $11(10)]\}$. These types of interference produce noisy data in remote sensing images and result in the presence of random noise and along the track dark stripes \{Fig. 1(a) [band 201 (5)]\} and bright stripes \{Fig. 1(b) [band 117 (12)]\} with random segment lengths [Figs. 1(a) and 1(b)]. Typical examples of random noise, abnormal columns, and vertical stripes present in L1R Hyperion images can be seen in Fig. 1.

Images in Figs. 1(a) and 1(b) are presented as examples of various bands with different types of random noise and stripes in Hyperion images from the Udaipur area, India, and the Lulea area, Sweden, respectively. Two types of vertical stripes can be distinguished in the Hyperion images of the study area after standard "Environment for Visualizing Images" (ENVI $\left.{ }^{\mathrm{TM}}\right)$ preprocessing for removing abnormal pixels $(-32,767)$, random noise \{Fig. 1(a) [band 121 (4), band 224 (6)]\}, abnormal columns \{Fig. 1(a) [band 201 (5)], Fig. 1(b) [band 117 (12), band 118 (13)]\}, and striping effect: global vertical stripes \{Fig. 1(a) [band 8 (1), band 121(3)], Fig. 1(b) [band 8 (8), band 11(10)] \} and local vertical stripes \{Fig. 1(a) [band 8 (2), band 224 (7)], Fig. 1(b) [band 8 (9), band $11(11)]\}$. Detection and reduction of the salt-and-pepper noise and correction of vertical stripes are essential for further processing.

\section{Previous Works on Noise Reduction and Correction from Hyperspectral Images}

A variety of algorithms has been proposed for the reduction of salt-and-pepper noise. The most widely applied approaches include median filtering, morphological filtering, contra-harmonic mean filtering, principal component analysis (PCA), minimum noise transformation (MNF)based method ${ }^{8,11}$ or interference and noise-adjusted PCA, ${ }^{12}$ spectral-spatial adaptive total variation modeling, ${ }^{13}$ linear regression, ${ }^{14}$ rak-1 tensor decomposition, ${ }^{15}$ spectral-spatial kernel regularization, ${ }^{16}$ and fast Fourier transformation (FFT), though FFT filtering assumes regular, ideally periodic signals in spectral data. These spectral data typically show almost arbitrary shapes, which are not very well handled by FFT. Therefore, this is not commonly applied to hyperspectral remote sensing datasets. ${ }^{17}$ Spatial interpolation using mean and median ratiobased approaches have been developed for automatic identification and correction of bad pixels with nonuniform noise in spatially uniform hyperspectral, multispectral, and panchromatic imagery ${ }^{18,19}$ An adaptive filtering by generalizing rational Laplacian pyramid was developed to reduce signal-independent noise from multiresolution images in order to increase the signal-tonoise ratio (SNR). ${ }^{20}$ Further, an MNF transformation-based algorithm has been developed to reduce signal-dependent noise from hyperspectral images using a parametric model to estimate noise from image scenes. ${ }^{21}$ 
There are several published algorithms for noise correction of vertical stripes (or destriping), which can be grouped into three groups: (1) frequency domain-based algorithms, (2) linear transformation-based algorithms, and (3) spatial domain-based algorithms. The recent studies on destriping on remote sensing data are explained below.

In frequency-domain filtering, stripe-related frequencies are estimated from noisy images using Fourier spectra and designing band-pass filters accordingly for denoising images. However, segregation of stripe-related frequencies is generally difficult to achieve, and therefore, signals with nonstripes-related features could be filtered out along with the stripes and thus could result in the loss of useful information. ${ }^{22-24}$ Wavelet analysis is also employed. The scaling and directional properties are used to detect stripe-related frequencies and thus used to remove stripes from remote sensing images. ${ }^{22,25,26}$ Some commercially available tools use FFT for removing vertical stripes (e.g., ERDAS Periodic Noise Removal or EPNR). ${ }^{13,24,27}$ Similarly, wavelet Fourier adaptive filtering ${ }^{26,28}$ is also implemented in some commercial software to correct nonperiodic image stripes.

Linear transformation-based algorithms are either eigen-space-based or tensor-based. ${ }^{14}$ Eigen-space-based algorithms include principal component (PC) transformations, MNF transformations, ${ }^{11,12}$ and tensor decompositions based on tensor analysis ${ }^{15}$ for noise reduction algorithms.

In the spatial domain, four main approaches have been described in the literature for destriping from remote sensing images: (1) low-pass filtering, (2) dark-current (DC) bias correction, (3) histogram matching, and (4) moment matching. The recent studies based on these approaches are explained here.

The low-pass spatial filters are the simplest spatial domain-based algorithms for destriping Hyperion data. In low-pass filtering, mean or median filter-based algorithms are mainly used where the radiance values of abnormal pixels from stripes are substituted by the median or mean radiance value from nonstripes adjacent neighboring normal pixels. However, there is a risk that high-frequency useful information may be removed using low-pass filters. The available "ENVI-SPEAR" and Flag Mask Correction available in the ENVI-plugin "Hyperion Tools" are based on the low-pass filtering algorithm.,

Several factors may lead to vertical stripes in remote sensing images, including nonlinearities and heating effects resulting from abnormal responses of a few detectors, and/or shift in the slit with respect to the focal plane. ${ }^{29}$ Assuming the above factors for stripes in remote sensing images, the DC bias-based correction algorithms have been developed. These algorithms assume that in pushbroom scanner systems, all cross-track detectors have similar statistical responses and cover similar materials along a flight line. Therefore, each detector has similar statistical responses and thus similar radiometric responses. Hence, a simple DC bias of the detectors across the direction of the detector array in pushbroom scanners may lead to striping in the images. Based on the above concept, Kruse ${ }^{30}$ and Kruse et al. ${ }^{29}$ have demonstrated and applied the techniques on airborne visible/infrared imaging spectrometer and Hyperion data, respectively. They suggested that a simple compensation per-column DC offset is appropriate to correct the striping effect. This technique provides destriped hyperspectral images, but the technique alters all the image pixels' values with respect to global band average and a column's average, regardless of whether pixels belong to stripes or nonstripes. ENVI's "General Purpose UtilitiesDestripe" is based on the above algorithm. Originally, the tool was designed for destriping horizontal images [e.g., Landsat Multispectral Scanner (MS)]. Hence, for destriping vertical images such as Hyperion, prior to the destriping procedure, the data should be rotated by $90 \mathrm{deg} .{ }^{6}$ Other concepts of striping effects in remote sensing images are assumed to be time variant and the linear detector's response. ${ }^{31}$ Consequently, modifications and improper calibration in offsets and gain values in one and/or more detectors result in striping in the images. Therefore, if the responses of all the detectors are appropriately calibrated, then their statistical distribution, such as means and variances, has similar responses and thus their statistical moments should match. Hence, columns from the noisy detectors can be rectified by constraining the statistical moments of columns to match the reference column's statistical moments from a properly calibrated detector. This approach has been introduced and applied by Datt et al. ${ }^{8}$ and Li et al. ${ }^{5}$ using global as well as local image average and variance as the reference moment for destriping Hyperion images. Similar algorithms have also been described by Lu et al., ${ }^{32}$ Xie et al., ${ }^{33}$ Acito et al., ${ }^{34}$ 
and Sheng and Zhang ${ }^{9}$ to destripe Hyperion images. Further, a linear least squares regression and global as well as local moment matching were used in the spectral domain by Adler-Golden et al. ${ }^{4}$ for destriping Hyperion images. The basic assumption of moment matching-based method for destriping is that the probability distributions of data at each column of the image should equal or match the probability distributions of the reference. Consequently, this approach assumes that parts of an image contain a complete statistical similarity to the whole image. Horn and Woodham ${ }^{31}$ presented a moment matching-based algorithm to remove stripes from Landsat MS images. This method is effective only in the case when the entire image scene is covered with an analogous material composition. However, artifacts may be introduced in the destriped images when using this method if the scene contains natural land use or land cover variations over and across the area. Similar techniques have been used by Dykstra and Segal ${ }^{35}$ in airborne imaging spectrometer data and Wegener ${ }^{36}$ on multisensor's data to remove stripes.

In this paper, we have reviewed previous studies for random noise reduction and stripes correction from remote sensing images. Previous literature as well as our experiment and examination show that there are no-data and invalid data pixels, random noise, and stripes present in Hyperion images. From our experiment, we found that invalid data pixels and random noise present in images should be removed or rectified before stripes correction from Hyperion images. Without these corrections before destriping, artifacts are created in the destriped images. These types of corrections have not been considered in previous studies. Therefore, we developed a better sequential procedure to remove no-data and invalid data pixels correction, random noise correction, and finally band columns and global and local stripes correction from Hyperion images.

\section{Study Area and Dataset}

Two areas have been selected in this study. One study area is close to the city of Udaipur, Rajasthan state in western India and another is close to the city of Luleå, Norrbotten County, Sweden. The developed algorithm has been applied on the Hyperion datasets from both study areas. The Udaipur scene was captured on 19 January 2004 and the location falls between latitude $24^{\circ} 6^{\prime} 56^{\prime \prime} \mathrm{N}$ to $24^{\circ} 54^{\prime} 45^{\prime \prime} \mathrm{N}$ and longitude $73^{\circ} 32^{\prime} 51^{\prime \prime} \mathrm{E}$ to $73^{\circ} 48^{\prime} 38^{\prime \prime}$ E. The geographical characteristics of the area are sparse vegetation covers and dry and semiarid conditions. The area has significant bedrock exposures and a rugged terrain containing noticeable hills and valleys. The study area contains more abundant minerals, including dolomite, rock phosphate, calcite, talc (soapstone), and several ornamental stones. The Luleå scene was captured on 26 August 2013 and location falls between latitude $65^{\circ} 08^{\prime} 44^{\prime \prime} \mathrm{N}$ to $65^{\circ} 58^{\prime} 17^{\prime \prime} \mathrm{N}$ and longitude $22^{\circ} 08^{\prime} 23^{\prime \prime} \mathrm{E}$ to $21^{\circ} 59^{\prime} 13^{\prime \prime} \mathrm{E}$. The geographical characteristics of the area include part snow covers in winter and partial vegetation covers in summer with subarctic conditions with short, mild to warm summers and long, cold, snowy winters. This area also has significant bedrock exposure in summer with undulated topography. Geologically, the area comes under Fennoscandian Shield with diverse lithology and contains a variety of minerals, including schorl, quartz, bertrandite, sulfides, and potassium minerals.

The availability of globally high-resolution spaceborne hyperspectral data is very limited. NASA'S Hyperion aboard on EO- 1 satellite is the only source of globally available data with a spectral range of 0.4 to $2.5 \mu \mathrm{m}$ and bandwidth of $10 \mathrm{~nm}$. The spatial resolution is $30 \mathrm{~m}$ from the height of the satellite's orbit of $705 \mathrm{~km}$. The Hyperion scanner is a pushbroom instrument with a 7.5-km swath width. The SNR is variable and ranges from 40 for short wave infrared (SWIR) to 190 for visible and near-infrared (VNIR). ${ }^{29}$ The L1R product of Hyperion has 242 bands, among them only 196 are calibrated (8 to 57 for the VNIR and 79 to 224 for the SWIR) and the remaining 44 bands have poor signal due to their detectors' low responsivity. There are only 196 unique channels due to an overlap between the VNIR and the SWIR focal planes. The data details are given in Table 1.

\section{Proposed Methodology}

In the proposed algorithm, random noise and vertical stripes are removed separately in the following steps. The flowchart of the proposed algorithm is shown in Fig. 2. 
Pal, Porwal, and Rasmussen: Noise reduction and destriping using local spatial statistics...

Table 1 Study Area and Data Details.

\begin{tabular}{ll}
\hline \hline Sensor type & \\
\hline Altitude of the sensor & $705 \mathrm{~km}$ \\
Swath width & $7.5 \mathrm{~km}$ \\
Number of total spectral bands & 242 \\
Number of unique spectral bands and & 220,357 to $2576 \mathrm{~nm}$ \\
their spectral range & \\
Number of calibrated bands (L1R) & $196(8$ to 57 for VNIR and 79 to 224 for SWIR) \\
Size of pixel (spatial resolution) & $30 \mathrm{~m}$ \\
Bandwidth (spectral resolution) & $10 \mathrm{~nm}$ \\
SNR & $190: 40$ \\
Udaipur scene & \\
Location of image & $73^{\circ} 32^{\prime} 51^{\prime \prime} \mathrm{E}$ to $73^{\circ} 48^{\prime} 38^{\prime \prime} \mathrm{E}$ and \\
& $24^{\circ} 6^{\prime} 56^{\prime \prime} \mathrm{N}$ to $24^{\circ} 54^{\prime} 45^{\prime \prime} \mathrm{N}$ \\
Data acquisition date & $19 \mathrm{January} 2004$ \\
Luleå scene & $264^{\circ} \mathrm{August} 2013$ \\
Location of image & $25^{\circ} 08^{\prime} 23^{\prime \prime} \mathrm{E}$ to $21^{\circ} 59^{\prime} 13^{\prime \prime} \mathrm{E}$ and \\
Data acquisition date & \\
\hline \hline
\end{tabular}

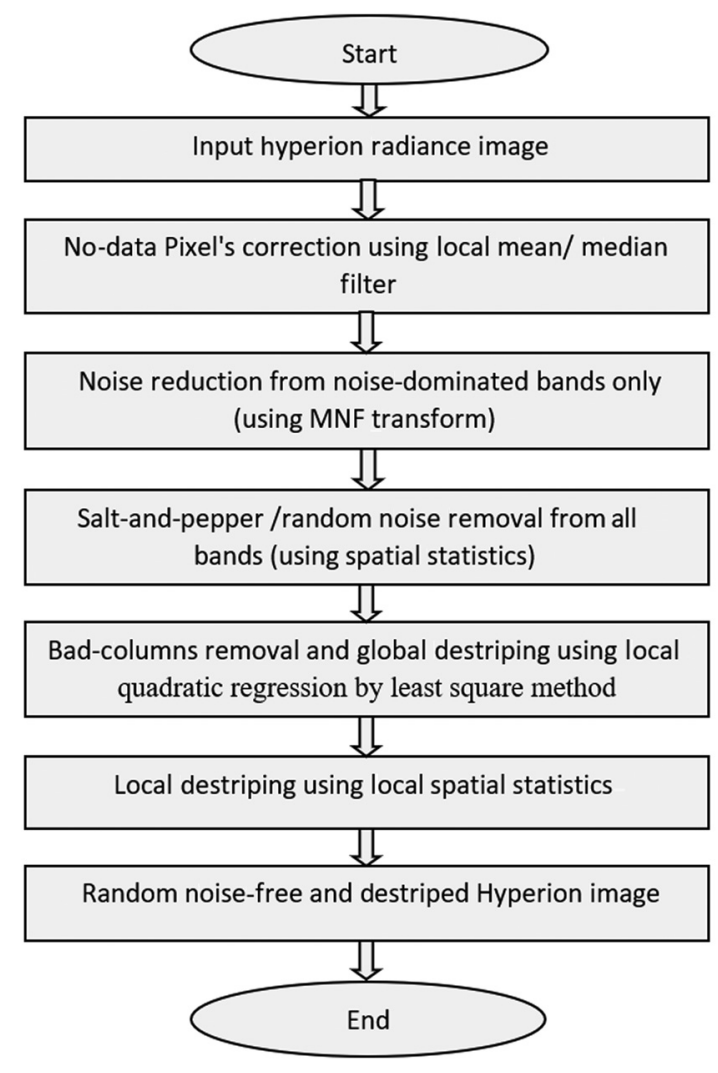

Fig. 2 Flowchart of the proposed methodology. 


\subsection{Pixel Correction with Null Values}

If the echo and smear correction process have not accounted for negative values, then small negatives such as $(-1)$ might be enlarged and documented as $-32,768 .^{2}$ Such negative values in Hyperion data show that the pixels do not hold any effective and valid data. Therefore, first the negative pixels' values from the whole image are detected and their values are set to zero, then the negative pixels' values are replaced and corrected by a neighboring mean value from a $(3 \times 3)$ median filter. The median filter with the smallest window size is used to preserve local characteristics so that pixels with negative values can be replaced with the average of nearest neighboring normal values and avoid oversmoothing and preserve pixels that contain actual zero values (e.g., water bodies in SWIR).

\subsection{Random/Salt-and-Pepper Noise Removal}

Random/salt-and-pepper noise is removed with the following two steps:

\subsubsection{MNF transformation}

The objective of this step is to correct only the noise-dominated bands without altering the coherent bands of a Hyperion image. This step is implemented as follows:

a. Identify and flag the noise-dominated bands $n_{j}$ from the Hyperion image $I$.

b. Carry out MNF transformation of $I$ to derive the transformed image $I_{t}$.

c. Select the noise-dominated MNF components $n_{t \_i}$ based on eigenvalues and perform inverse MNF transformation of $I_{t}$ after discarding $n_{t_{-} i}$ to obtain filtered image $I_{f}$.

d. Examine the flagged noise-dominated bands $n_{j}$ in $I_{f}$. Some of the bands $n_{j}$ would become coherent $\left(c_{j}\right)$ after step 5.2.1(c).

e. Iteratively implement steps 5.2.1(c) and 5.2.1(d) using lower and lower threshold eigenvalues for selecting noise-dominated MNF components until all original noise-dominated bands become coherent.

f. Replace the original noise-dominated bands $n_{j}$ by the now coherent bands $c_{j}$ in the original image $I$.

\subsubsection{Abnormal brightness correction}

The objective of this step is to identify, flag, and correct the pixels with abnormal brightness values compared to the brightness level of a local neighborhood. It is implemented as follows:

a. Apply a $9 \times 9$ moving window to the Hyperion image $I$ obtained after step 5.2.1 to generate three images: $I_{\text {Mean }}, I_{\text {StdDev }}$, and $I_{\text {Diff }}$. The image $I_{\text {Mean }}$ is obtained by replacing the central pixel value with the mean of the window. The image $I_{\text {StdDev }}$ is obtained by replacing the central pixel value with the standard deviation (STD) of the pixel from the mean of the $9 \times 9$ window. The image $I_{\text {Diff }}$ is obtained by calculating the absolute difference between the original image $I$ and the image $I_{\text {Mean }}$. A $9 \times 9$ window $\left(270 \times 270 \mathrm{~m}^{2}\right)$ size is used because it adequately represents the brightness of the local neighborhood of a pixel.

b. If the value of a pixel of $I_{\text {Diff }}$ is greater than or equal to three times the value of the image $I_{\text {StdDev }}$, then the pixel is flagged as a bad pixel and its value on the image $I$ is replaced by its corresponding value in the image $I_{\text {Mean }}$.

\subsection{Destriping}

Bad columns and vertical-stripes correction is implemented as follows.

\subsubsection{Correction of bad columns and global vertical stripes}

Bad columns and global vertical stripes are the stripes whose lengths run throughout the image length. 
The objective of this step is to identify and correct the brightness values of global vertical stripes by forcing their average brightness values to conform to the average of the neighboring columns. It is implemented as follows:

a. Calculate, for each band, the average brightness value of each column and draw a profile $\left(P_{\mathrm{CA}}\right)$ of the column averages across the band.

b. Estimate the maximum trough (or crest) width on $P_{\mathrm{CA}}$ in terms of the number of columns $\left(n_{c}\right)$.

c. A curve $\left(P_{\mathrm{FIT}}\right)$ is fitted to the column average profile $P_{\mathrm{CA}}$ using quadratic regression by a least squares method in a local moving window of $10 n_{c} \times 1$ size and replacing the average brightness value of the central column by the corresponding value obtained from quadratic regression by the least squares method.

The model used for curve fitting in this step is as described below:

$$
Y_{i}=a x_{i}^{2}+b x_{i}+c+\mathcal{E}_{i},
$$

where $a$ is the regression coefficient for the quadratic effect of $x$ on $Y, b$ is the regression coefficient for the linear effect of $x$ on $Y, c$ is the $Y$ intercept, and ${ }_{i}$ is the random error in $Y$ for observation $i$.

d. Subtract $P_{\mathrm{FIT}}$ from $P_{\mathrm{CA}}$ to calculate, for each column, the difference between the observed average brightness value and the expected brightness value based on the brightness values of the neighboring pixels.

e. The difference is added to each pixel of the respective column to correct the bad columns and global vertical stripes.

\subsubsection{Local destriping}

Local vertical stripes are the stripes whose lengths run randomly in a segment of the image length. Finally, the local vertical stripes are corrected by forcing the brightness value of the pixels to the same brightness level as that of the local neighborhood. The step is implemented through the following procedures.

a. Apply a $3 n_{c} \times 3 n_{c}$ [see step 5.3.1(b)] moving window to the corrected image $I_{\text {Corr }}$ obtained after step 5.3.1 to generate three images: $I_{\text {Corr_Mean }}, I_{\text {Corr_StdDev }}$, and $I_{\text {Corr_Diff }}$.

The image $I_{\text {Corr_Mean }}$ is obtained by replacing the central pixel value by the mean of the window. The image $I_{\text {Corr StdDev }}$ is obtained by replacing the central pixel value by the STD of the pixel from the mean of the window. The image $I_{\text {Corr_Diff }}$ is obtained by calculating the absolute difference between the original image $I$ and the image $I_{\text {Corr_Mean }}$.

b. If the value of a pixel of $I_{\text {Corr_Diff }}$ is greater than its corresponding value on the image $I_{\text {Corr_StdDev }}$, then the pixel is flagged as a bad pixel. If the number of bad pixels in a single column within the moving window is greater than $90 \%$ of the total number of pixels in the column, then the column is flagged as a local stripe and the values of the bad pixels are replaced by their corresponding values on $I_{\text {Corr_Mean }}$.

The above algorithm is applied in Hyperion data using the "Interactive Data Language" (IDL) program to computerize the noise and stripes correction procedure.

\section{Results and Discussion}

The above algorithm has been implemented and processed Hyperion images of the study areas using ENVI ${ }^{\mathrm{TM}}$ software and a programming language IDL provided by Exelis Visual Information Solutions. The effectiveness of the proposed algorithm and results were compared with the results obtained using some of the previous most widely used algorithms (low-pass filtering, ${ }^{7}$ DC bias correction, ${ }^{8}$ global and local moment matching, ${ }^{33}$ and local moment matching in spectral domain ${ }^{4}$ ) from Hyperion images from the study areas. To show the effectiveness and efficiency of the algorithm, bands 8, 57, 121, 180, and 224 of the Udaipur and Luleå Hyperion 
Pal, Porwal, and Rasmussen: Noise reduction and destriping using local spatial statistics...

images are selected to demonstrate the performance. Bands 8 and 57 fall within the VNIR region and are relatively noise-free, while bands 121,180 , and 224 are from the water vapor absorption range of the SWIR region and are extremely noisy (Figs. 3 and 4). The spectral profiles before and after noise removal are shown in Fig. 5. The proposed algorithm and the previous algorithms return similar results for the noise-free bands (e.g., bands 8 and 57 in Figs. 3 and 4). However, for noise-dominated bands (e.g., bands 121, 180, and 224 in Figs. 3 and 4), the proposed method returns significantly better results compared to the previous algorithms. These results show that it is critical to remove random noise before attempting destriping. Figure 5 compares the spectral radiance profiles from Hyperion images of the Udaipur area [pixel position $(1555,7)]$ and from the Luleå area [pixel position $(1555,145)]$ after applying the proposed algorithm vis-à-vis previous algorithms. The proposed algorithm generally preserves the original spectral features, but at the same time corrects the false absorption features and spikes [Figs. 5(a), 5(b), 5(a'), and 5(b')]. In Fig. 5(a'), band $178\left(x_{1}, y_{1}\right)$, and the corresponding spectra in Fig. 5(a) $\left(x_{1}, y_{1}\right)$,

(a)
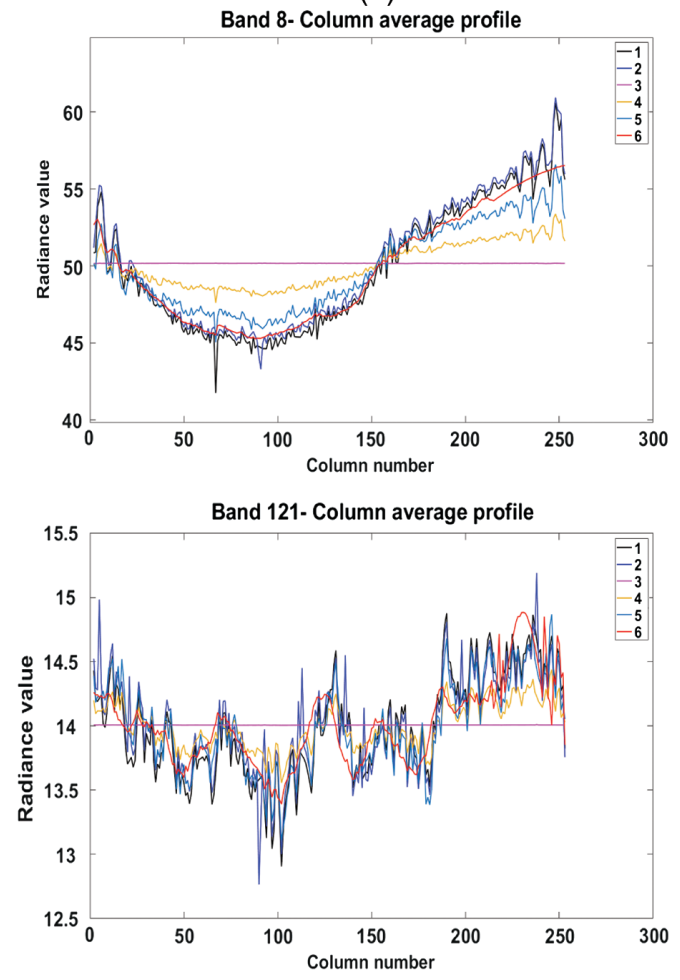

Band 224- Column average profile

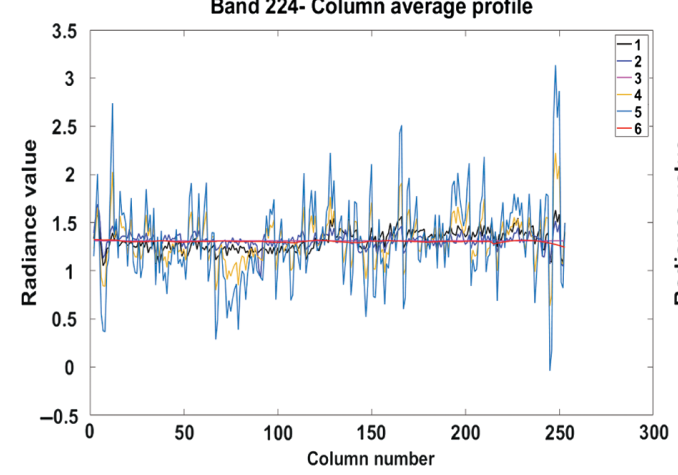

(b)
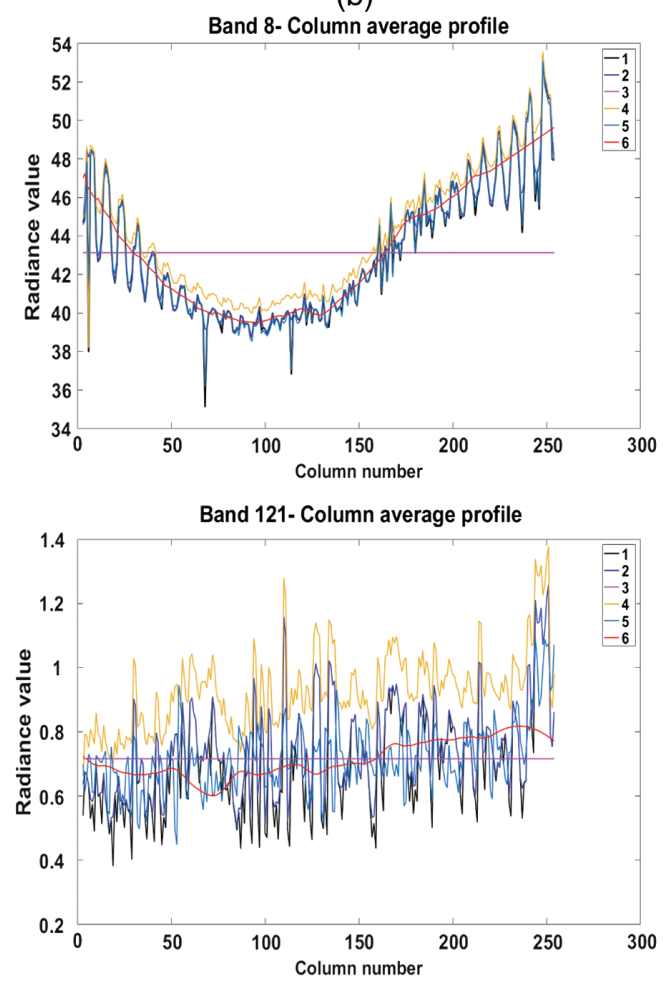

Band 224- Column average profile

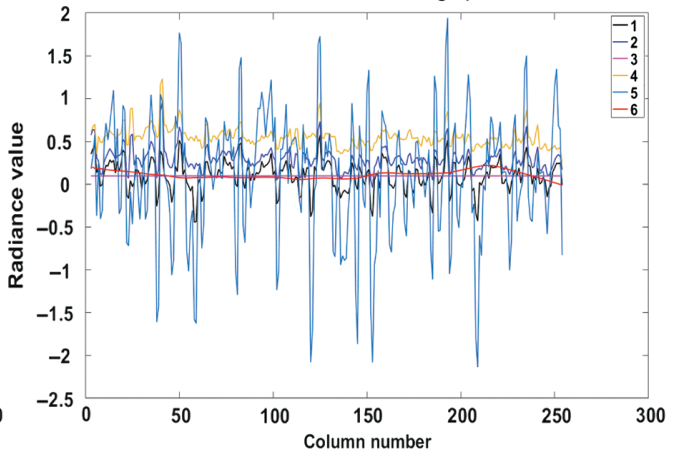

Fig. 3 Hyperion data from (a) the Udaipur scene and (b) the Luleå scene: Column-average profiles of bands 8,121 , and 224 before destriping (black) and after destriping using low-pass filtering ${ }^{16}$ (blue), DC bias correction ${ }^{37}$ (magenta), global and local moment matching ${ }^{11}$ (golden yellow), spectral local moment matching ${ }^{2}$ (deep sky blue), and local spatial statistics and local quadratic regression by least squares method (this paper; red). 
Band 8

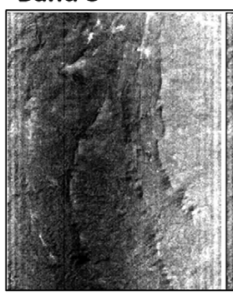

(1)

Band 57

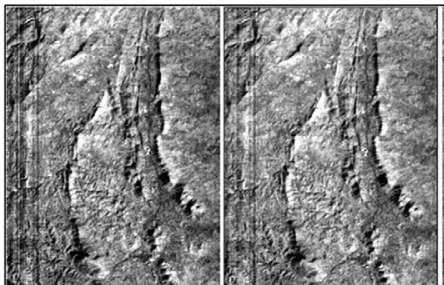

(1)

Band 121

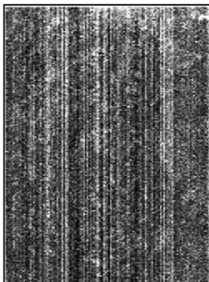

(1)
(2)

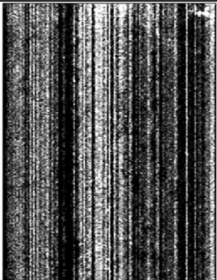

(2)

Band 180

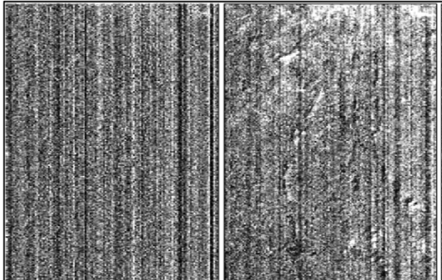

(1)

Band 224

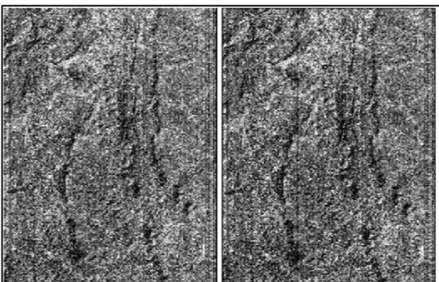

(1)
(2)

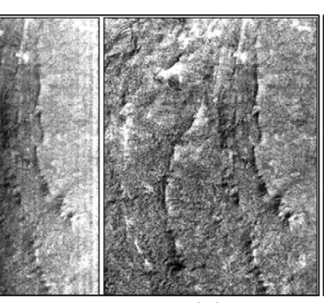

(3)

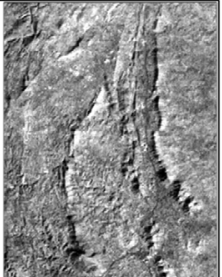

(3)

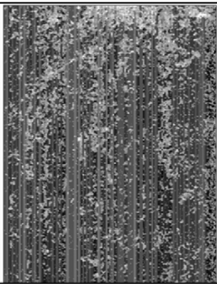

(3)

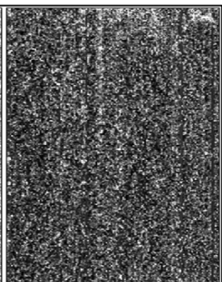

(3)

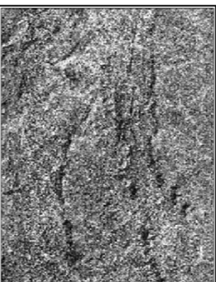

(3)

(a)

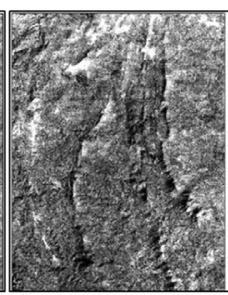

(4)

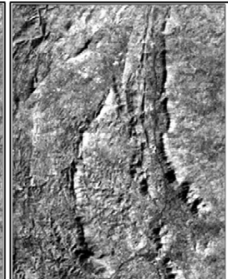

(4)

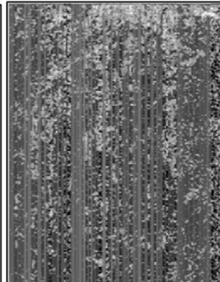

(4)

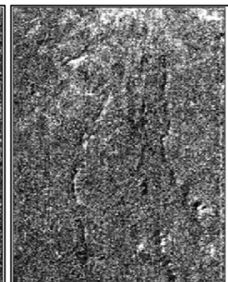

(4)

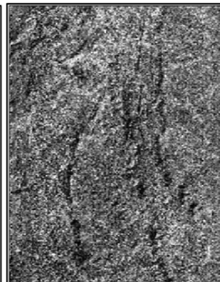

(4)

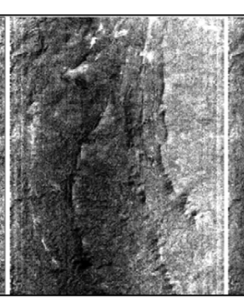

(5)

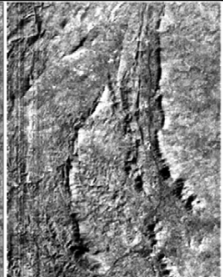

(5)

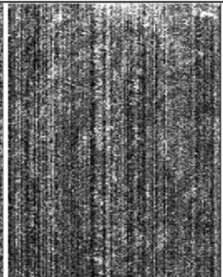

(5)

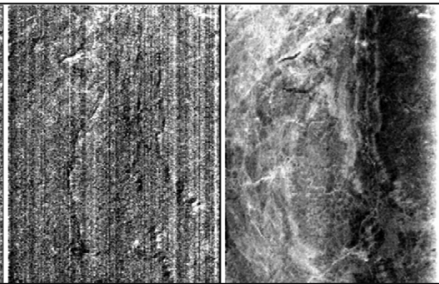

(5)

(6)

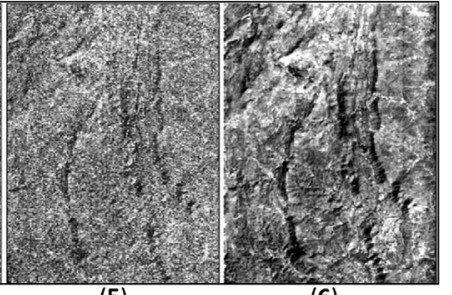

(5)

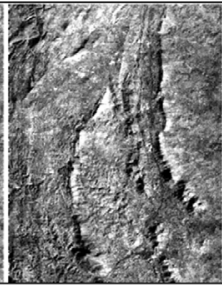

(6)

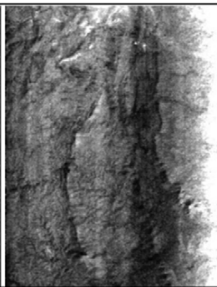

(6)

Fig. 4 Hyperion images before and after correction by various algorithms from (a) the Udaipur scene and (b) the Luleå scene: bands 8, 57, 121, 180, and 224 of the Hyperion images of the study areas, (1) before destriping and after destriping using (2) low-pass filtering, ${ }^{16}$ (3) DC bias correction, ${ }^{37}$ (4) global and local moment matching, ${ }^{11}$ (5) spectral local moment matching, ${ }^{2}$ and (6) local spatial statistics and local quadratic regression by least squares method (this paper).

the false absorption feature at $1931 \mathrm{~nm}$, and Fig. 5(b') band $97\left(x_{5}, y_{5}\right)$, and the corresponding spectra in Fig. 5(b) $\left(x_{5}, y_{5}\right)$, the false absorption feature at $1124 \mathrm{~nm}$, which are generated by a local stripe, are corrected by the proposed algorithm much better compared to the previous algorithms. Similarly, in Fig. 5(a'), band $219\left(x_{4}, y_{4}\right)$ and the corresponding spectra in Fig. 5(a) $\left(x_{4}, y_{4}\right)$, the false absorption feature at $2345 \mathrm{~nm}$, and in Fig. 5(b') band $122\left(x_{8}, y_{8}\right)$ and the corresponding spectra in Fig. 5(b) $\left(x_{8}, y_{8}\right)$, the false absorption feature at $1366 \mathrm{~nm}$, which are generated by salt-and-pepper noise, are corrected by the proposed algorithm, whereas the 
(b)

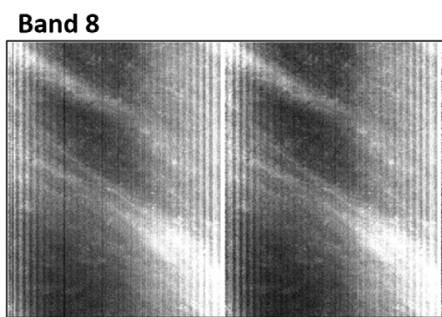

(1)

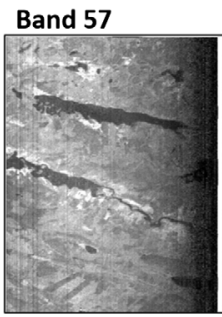

(1) Band 121

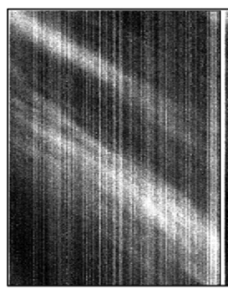

(1) Band 180

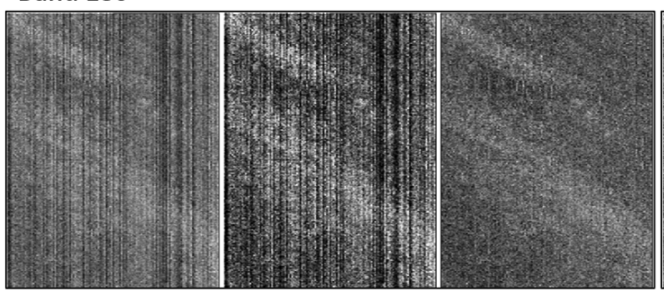

(1)

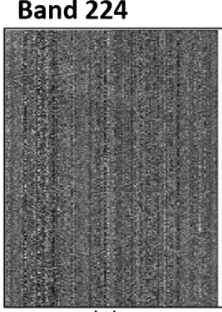

(1)
(2)

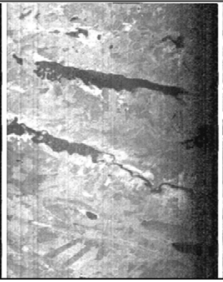

(2)

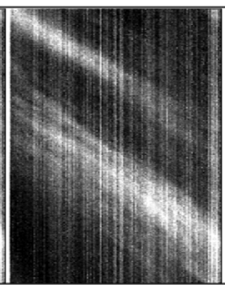

(2)

(2)

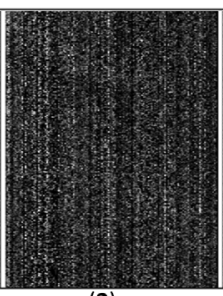

(2)

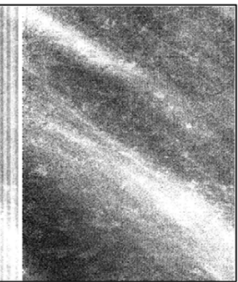

(3)

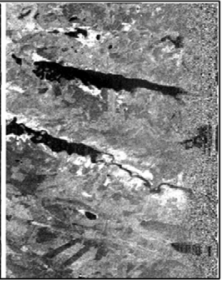

(3)

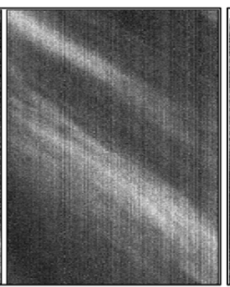

(3)

(3)

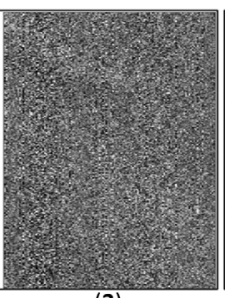

(3)

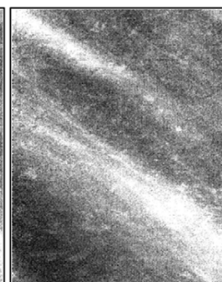

(4)

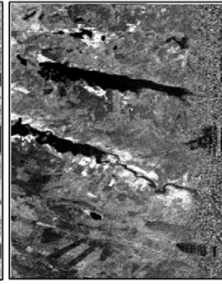

(4)

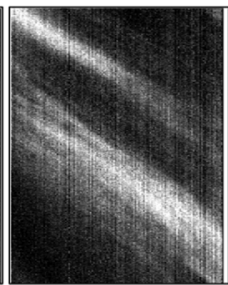

(4)

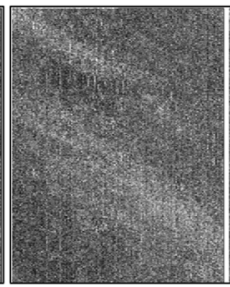

(4)

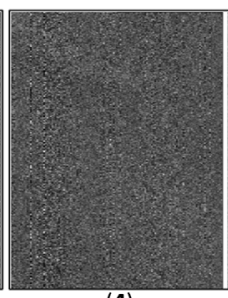

(4)

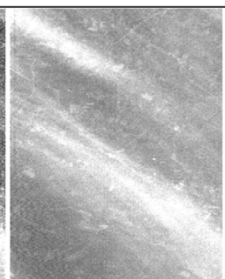

(5)

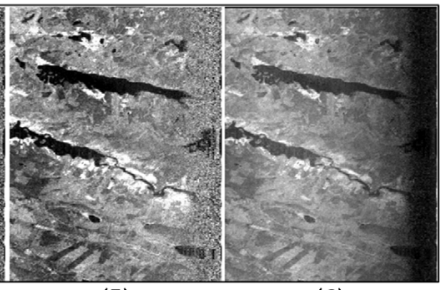

(5)

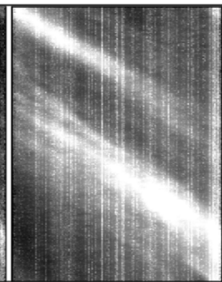

(5)

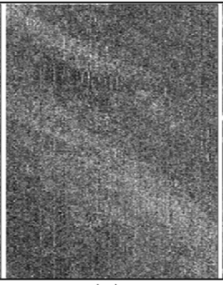

(5)

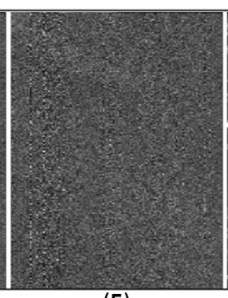

(5)

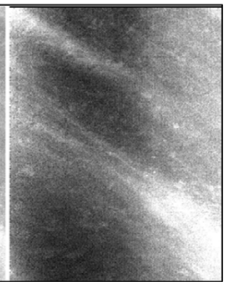

(6)

(6)

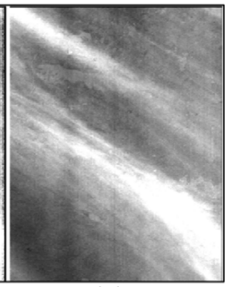

(6)

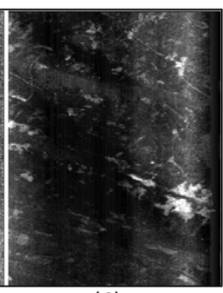

(6)

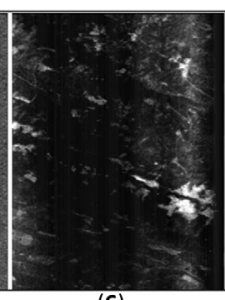

(6)

Fig. 4 (Continued)

previous algorithms fail to correct it. False absorption features due to global vertical stripes and bad columns are corrected equally effectively by all algorithms [e.g., false absorption features at 2153 and $2163 \mathrm{~nm}$ in Fig. 5(a), $\left(x_{2}, y_{2}\right)$ and $\left(x_{3}, y_{3}\right)$ and the corresponding band image in Figs. 5(a') band $200\left(x_{2}, y_{2}\right)$ and band $201\left(x_{3}, y_{3}\right)$, and false absorption features at 1316 and $1326 \mathrm{~nm}$ in Fig. 5(b) $\left(x_{6}, y_{6}\right)$ and $\left(x_{7}, y_{7}\right)$ and the corresponding band image in Figs. 5(b') band $117\left(x_{6}, y_{6}\right) \&$ band $\left.118\left(x_{7}, y_{7}\right)\right]$. However, compared to the previous algorithms, the proposed algorithm preserves true absorption features much more effectively [e.g., Fig. 5(a), absorption features at 2012 and $2345 \mathrm{~nm}$; Fig. 5(b), absorption features at 1215 and $1316 \mathrm{~nm}$ ].

The false absorption feature at 2153 to $2163 \mathrm{~nm}$ in Fig. 5(a) $\left(x_{2}, y_{2}\right)$ and $\left(x_{3}, y_{3}\right)$ and the corresponding images Figs. 5(a') band $200\left(x_{2}, y_{2}\right)$ and band $201\left(x_{3}, y_{3}\right)$ is from a striped region [pixel position $(1555,7)$ ], while the true absorption feature on radiance spectra at 2153 to 
Pal, Porwal, and Rasmussen: Noise reduction and destriping using local spatial statistics...

(a)

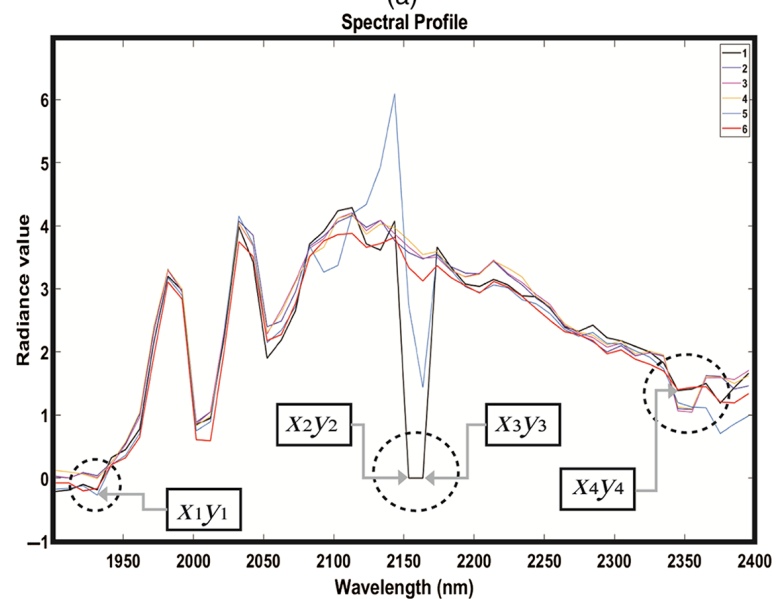

(b)

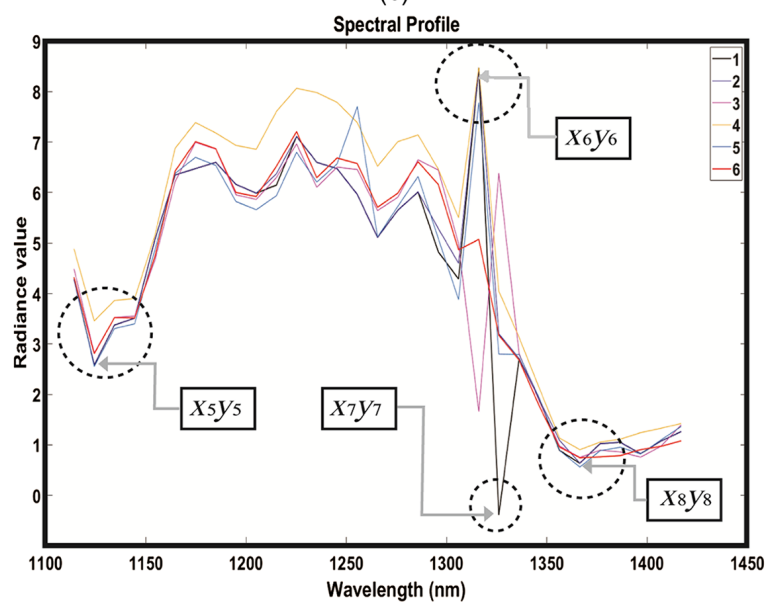

(a')
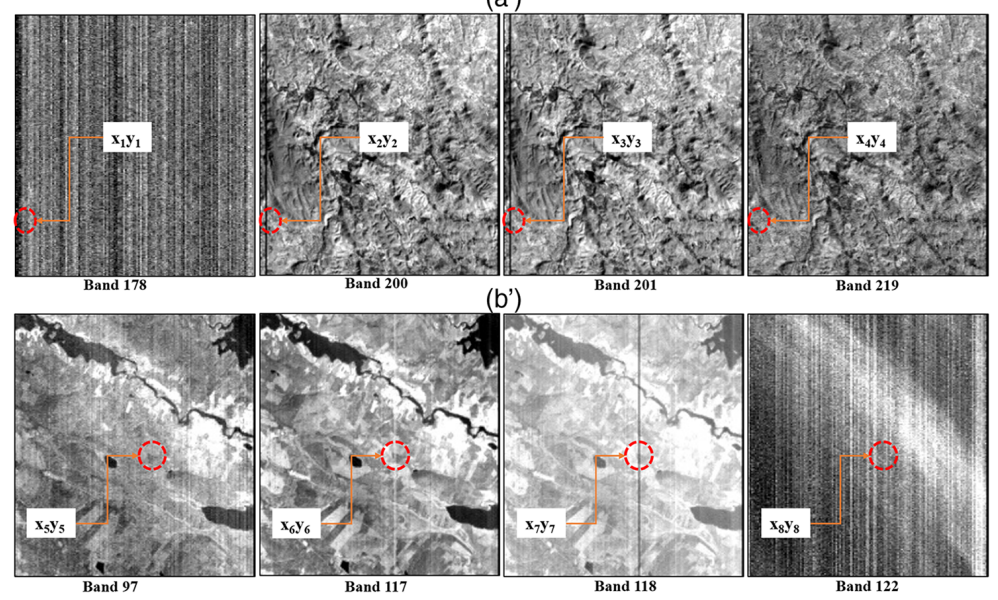

Fig. 5 Spectral profile of Hyperion radiance data-original spectrum before destriping (black) and corrected spectrum after destriping using low-pass filtering ${ }^{16}$ (blue), DC bias correction ${ }^{37}$ (magenta), global and local moment matching ${ }^{11}$ (golden yellow), spectral local moment matching ${ }^{2}$ (deep sky blue), and local spatial statistics and local quadratic regression by least squares method (this paper; red). (a) Spectral profile of Hyperion data from the Udaipur scene in spectral range of 1900 to $2395 \mathrm{~nm}$ from pixel position $(1555,7)$ and (b) spectral profile of Hyperion data from the Luleå scene in spectral range of 1115 to $1425 \mathrm{~nm}$ from pixel position $(1555,145)$. (a') Pixel location in various bands of Hyperion image from Udaipur scene corresponding to the spectra (a) and (b') pixel location in various bands of Hyperion image from Luleå scene corresponding to spectra (b). Please note that $(x, y)$ shows the corresponding spectral position between spectral profiles $(a, b)$ and bands in images (a', b'). 
$2163 \mathrm{~nm}$ in a nonstriped region [position $(1555,10)$ ] close to a vertical stripe shows similar absorption feature as the corrected spectra by the proposed method. The proposed method preserves the true absorption feature, while the previous algorithms may mar the true absorption feature of the spectra.

In the above results, the effectiveness of the proposed algorithm is shown and compared with the most widely used techniques for random noise reduction and destriping from hyperspectral images. The above approaches are applied on two Hyperion images from different geographical locations [(a) Udaipur, India (subtropical and semi-arid) and (b) Luleå, Sweden (temperate and cold)] to demonstrate the generalized application of the proposed method to hyperspectral images (Hyperion) from any location on the globe. In Fig. 3, column average profile before and after destriping from three bands (bands 8, 121, and 224) of Hyperion images from Udaipur (a) and Luleå (b) are shown to explain the disparity of the proposed and other algorithms with respect to the original data column average profile while noise reduction is due to destriping. The column average profiles from all bands [band 8 (initial), band 121 (middle), and band 224 (last)] show that the proposed algorithm mimics and smooths the original column average profiles, whereas other algorithms deviate. The columns' average profiles shown in Figs. 3(a) and 3(b) show that the original geospatial data of the images are preserved by the proposed method, while other algorithms significantly alter the original data. In Fig. 4, two images [Udaipur (a) and Luleå (b)], five bands (bands 8, 57, 121, 180, and 224), two bands from VNIR and three bands from SWIR, among them two bands from the near water absorption region, have been chosen to represent the complete band scenario of the Hyperion images. Figure 4 represents bands 8 and 57 from the VNIR region of Hyperion images from both the Udaipur scene and the Luleå scene indicating that all algorithms produce stripes-free bands, whereas stripes in random noise-dominated bands [e.g., Figs. 4(a) and 4(b) bands 121, 180, and 224] the proposed algorithm produces improved results whereas other algorithms create artifacts in the image while destriping. Figures 5(a') and 5(b') original bands from Udaipur (a') and Luleå (b') Hyperion images and corresponding spectra from the original images and after destriping, shown in Figs. 5(a) and 5(b), reveal that the proposed algorithm produces improved spectra while preserving the original spectral information compared to other techniques. Based on the above results from Figs. 3-5, it is clear that the proposed algorithms rectify the Hyperion images from random noise and striping effects more robustly compared to other algorithms while preserving the original data information.

Further, statistical evaluation by using series of image quality indices have been applied on the Hyperion dataset to evaluate the performance of the proposed algorithm vis-à-vis some recently published algorithms. The image quality indices include mean, ${ }^{38,39} \mathrm{STD},{ }^{38,39}$ mean relative deviation (MRD), ${ }^{9}$ distortion of edge radiance (DER), ${ }^{38,39}$ distortion of gain adjustment (DGA), ${ }^{38,39}$ noise reduction ratio (NRR), ${ }^{9,38-41}$ improvement factor (IF) of radiometric quality, ${ }^{39,42}$ Shannon entropy (SNEP), ${ }^{38,39}$ and SNR, ${ }^{37,38,39,43}$ (Table 2). The objective of these analyses is to select the best statistical-based method for destriping Hyperion data.

Mean reflects the general radiation level of the image and STD reflects the general variability radiation across the image. ${ }^{38,39}$ The change of mean and STD after destriping indirectly reflects the degree of preservation of the original image information, ${ }^{39}$ in which the less the change, the better the destriping effect. ${ }^{39}$ The change of the image mean is minimum for the proposed algorithm. The change in the image STD is similar for all algorithms.

MRD is used to evaluate the effectiveness of an algorithm in retaining the original information content of the image. It is calculated as

$$
\mathrm{MRD}=\frac{1}{\mathrm{MN}} \sum_{i=1}^{\mathrm{MN}} \frac{\left|\widehat{z_{i}}-g_{i}\right|}{g_{i}} \times 100 \%,
$$

where $g_{i}$ is the pixel value of the original image, $\widehat{z_{i}}$ is the pixel value of the destriped image, and $\mathrm{MN}$ is the total number of pixels. MRD represents and characterizes the distortion of the original image caused by the adopted destriping method. ${ }^{9}$ Lower values of MRD indicate less distortion of the original image. ${ }^{9}$ The MRD value for the proposed algorithm is smaller than all previous algorithms, except the global and local moment matching. ${ }^{8}$ 
Pal, Porwal, and Rasmussen: Noise reduction and destriping using local spatial statistics...

Table 2 Image Quality Indices of Hyperion Images.

\begin{tabular}{|c|c|c|c|c|c|c|c|c|}
\hline \multirow[b]{2}{*}{ Method } & \multicolumn{8}{|c|}{ Statistical test } \\
\hline & Mean STD & MRD (\%) & DER & DGA & NRR & IF & SNEP & SNR \\
\hline \multicolumn{9}{|c|}{ A. Summary of average image quality indices from all bands } \\
\hline \multicolumn{9}{|c|}{ Hyperion image from Udaipur } \\
\hline Original data & 8.073211 .191 & - & 87.286 & 6.204 & - & - & 3.076 & 10.501 \\
\hline \multicolumn{9}{|c|}{ Destriped image obtained using } \\
\hline Low-pass filtering $^{7}$ & 8.14225 .799 & 0.706 & 3.201 & 6.052 & 1.093 & -0.003 & 2.917 & 14.812 \\
\hline DC bias correction ${ }^{8}$ & 8.14225 .256 & 1.689 & 3.199 & 3.537 & 1.110 & -5.212 & 3.079 & 14.93 \\
\hline Global and local moment matching ${ }^{33}$ & 8.14325 .257 & 1.583 & 3.199 & 4.303 & 0.999 & -4.343 & 2.812 & 14.927 \\
\hline Local moment matching ${ }^{4}$ & 8.073211 .180 & 5.408 & 87.286 & 1.131 & 0.923 & 0.741 & 3.116 & 14.613 \\
\hline Proposed method & 8.07335 .169 & 1.678 & 2.677 & 0.667 & 1.127 & 0.731 & 3.183 & 16.036 \\
\hline \multicolumn{9}{|c|}{ Hyperion image from Luleå } \\
\hline Original data & 6.293911 .166 & - & 71.336 & 10.495 & - & - & 3.469 & 13.558 \\
\hline \multicolumn{9}{|c|}{ Destriped image obtained using } \\
\hline Low-pass filtering $^{7}$ & 6.294011 .160 & 0.5436 & 71.341 & 10.386 & 0.999 & -0.007 & 3.385 & 13.678 \\
\hline DC bias correction ${ }^{8}$ & 6.293610 .688 & 1.7564 & 71.336 & 8.812 & 0.357 & -2.027 & 3.722 & 13.694 \\
\hline Global and local moment matching ${ }^{33}$ & 6.29376 .047 & 1.6065 & 5.184 & 15.998 & 0.863 & 6.218 & 3.658 & 13.966 \\
\hline Local moment matching ${ }^{4}$ & 6.293811 .166 & 3.5353 & 71.336 & 10.489 & 1.001 & -2.754 & 3.722 & 13.566 \\
\hline Proposed method & 6.29826 .813 & 1.485 & 5.121 & 10.071 & 1.175 & 6.342 & 3.754 & 14.154 \\
\hline
\end{tabular}

B. Summary of average image quality indices from water absorption bands (bands 120 to 132 and 165 to 182)

\begin{tabular}{|c|c|c|c|c|c|c|c|c|c|}
\hline \multicolumn{10}{|c|}{ Hyperion image from Udaipur } \\
\hline Original data & 1.0486 & 5.688 & - & 16.022 & 0.065 & - & - & 3.075 & 1.420 \\
\hline & \multicolumn{9}{|c|}{ Destriped image obtained using } \\
\hline Low-pass filtering $^{7}$ & 1.0316 & 0.679 & 8.982 & 0.067 & 0.059 & 1.001 & 3.386 & 2.254 & 1.855 \\
\hline DC bias correction ${ }^{8}$ & 0.8316 & 0.626 & 8.972 & 0.257 & 2.370 & 0.611 & 3.422 & 3.120 & 1.901 \\
\hline Global and local moment matching ${ }^{33}$ & 0.8416 & 0.706 & 9.851 & 0.068 & 1.310 & 0.603 & 3.324 & 2.490 & 1.910 \\
\hline Local moment matching ${ }^{4}$ & 0.8356 & 5.669 & 10.910 & 15.988 & 0.047 & 1.001 & 3.738 & 3.163 & 1.349 \\
\hline Proposed method & 1.1723 & 1.217 & 2.345 & 0.007 & 0.001 & 1.563 & 24.968 & 3.512 & 3.119 \\
\hline \multicolumn{10}{|c|}{ Hyperion image from Luleå } \\
\hline Original data & 0.510 & 7.043 & - & 24.795 & 0.026 & - & - & 3.268 & 1.339 \\
\hline \multicolumn{10}{|c|}{ Destriped image obtained using } \\
\hline Low-pass filtering $^{7}$ & 0.511 & 7.051 & 0.455 & 24.799 & 0.022 & 0.999 & -0.002 & 3.024 & 1.711 \\
\hline DC bias correction ${ }^{8}$ & 0.504 & 7.042 & 3.545 & 14.796 & 7.596 & 0.310 & -2.086 & 3.683 & 1.521 \\
\hline Global and local moment matching ${ }^{33}$ & 0.643 & 0.424 & 2.906 & 0.072 & 0.032 & 0.783 & 58.387 & 3.557 & 2.672 \\
\hline Local moment matching ${ }^{4}$ & 0.509 & 7.043 & 4.010 & 24.796 & 0.016 & 1.001 & -2.205 & 3.683 & 1.374 \\
\hline Proposed method & 0.612 & 1.349 & 0.710 & 0.054 & 0.005 & 1.119 & 61.271 & 3.773 & 3.757 \\
\hline
\end{tabular}

Note: The results of the proposed method are highlighted in bold. STD, standard deviation; MRD, mean relative deviation; DGA, distortion of gain adjustment; DER, distortion of edge radiance; SNEP, Shannon entropy; IF, Improvement Factor; SNR, signal-to-noise ratio; NRR, noise reduction ratio. 
DER is the variance of the mean value vector in the row direction of the image and reflects the degree of irregularity of radiation in the scan-line direction. ${ }^{38}$ As compared to the previous algorithms, the proposed algorithm produces minimum DER.

DGA is the variance of the mean value vector in the column direction and is a measure of the degree of irregularity of radiation in the direction of the flight. ${ }^{38}$ The lower the DGA values, the less the distortion. ${ }^{38}$ The DGA value after destriping is minimum for the proposed algorithm.

NRR is achieved by an algorithm. It is estimated through the averaged power spectrum down the columns of an image, $P_{i}(D)$ (where $D$ is the distance from the origin in Fourier space). The noise components of the spectrum, $N_{i}$, are defined as ${ }^{9,40}$

$$
N_{i}=\sum_{\mathrm{BW}_{n}} P_{i}(D),
$$

where $\mathrm{BW}_{n}$ is the noisy region of the image spectrum. The NRR for a destriped image $N_{k}$ is defined as ${ }^{9,40}$

$$
N R R=\frac{N_{O}}{N_{k}},
$$

where subscript $N_{O}$ is the original image. Ideally, destriping would lead to increased noise reduction and hence high NRR. ${ }^{39,41}$ The proposed algorithm returns the highest NRR, thus indicating the maximum reduction of noise.

IF has been used to measure the improvement in radiometric quality of an image after applying destriping algorithms. ${ }^{39,42}$ IF is calculated and evaluated as

$$
\begin{aligned}
& d_{R}(j)=M_{\mathrm{IR}}(j)-M_{I}(j), \\
& d_{E}(j)=M_{\mathrm{IE}}(j)-M_{I}(j),
\end{aligned}
$$

where $j$ is the line number; $M_{\mathrm{IR}}(j)$ is the cross-track profile mean radiance of the raw image; $M_{\mathrm{IE}}(j)$ is the cross-track profile mean radiance of the destriped image; and $M_{\mathrm{I}}(j)$ is the mean radiance of the image obtained by low-pass filtering from cross-track profile.

Then, $\mathrm{IF}^{39,42}$ is defined as

$$
\mathrm{IF}=10 \log _{10}\left[\frac{\sum_{j} d_{R}^{2}(j)}{\sum_{j} d_{E}^{2}()}\right]
$$

High IF values indicate substantial improvement in the radiometric quality of the image. ${ }^{39,42}$ Again, the IF is second highest for the proposed algorithm after the local moment matching algorithm. ${ }^{4}$

SNEP is one way to represent information entropy, which measures the richness of the information quantity in an image. ${ }^{38,39}$ It is calculated using the following equation: ${ }^{38,39,44}$

$$
H=-\sum_{i=1}^{M} P_{i} \log P_{i}
$$

In the above equation, $i$ and $P_{i}$ denote the image's gray value and the probability of gray value $I$, respectively. Enhancement of SNEP values indicates preservation and improvement of the information content of an image. ${ }^{38,39,44}$ Again, the SNEP value for the proposed algorithm is higher as compared to the previous algorithms.

SNR is defined as the ratio of maximum signal strength to the background noise present in an image. ${ }^{37,43}$

A local variance method is used to estimate SNR using the following formula:

$$
\mathrm{SNR}=20 \log _{10} \times\left(\frac{\mathrm{Mean}_{H}}{\mathrm{RSM}_{\text {error }}}\right),
$$


where $\mathrm{Mean}_{H}$ and $\mathrm{RSM}_{\text {error }}$ are the average signal value and square root of the mean of variance, respectively, of a completely homogenous portion of the image.

High SNR indicates less distortion and enhanced signal information. Table 2 shows that the destriped image obtained by applying the proposed algorithm has the highest SNR.

The above statistical parameters are separately calculated from all the bands [Table 2(A)] and water absorption bands [Table 2(B)] from the Udaipur scene and the Luleå scene and are shown in Table 2. All the statistical tests from all bands reveal that the proposed algorithm reflects an improved image quality compared to other algorithms. Further, image quality indices show that the water absorption bands improved significantly by the proposed method compared to other algorithms.

The ultimate goal of this work is to develop a method to effectively reduce random and pattern noise or spatial nonuniformity in Hyperion images while considering the least or no alteration of the normal data values in the corrected image with respect to the original image. It is evident from Figs. 3-5 and Table 2 that the proposed method corrects random noise, bad columns, and stripes effectively and produces noise-free and information-rich Hyperion images.

\section{Summary and Conclusions}

Random noise, bad columns, and striping in Hyperion images produce false spectral absorption features that mislead the results in natural resources assessments. Therefore, noise reduction and destriping from Hyperion images are essential before evaluations of natural resources assessments from the same. Therefore, we propose a method to reduce random noise and remove bad columns and vertical stripes from Hyperion images based on local spatial statistics and local quadratic regression by least squares method. The proposed algorithm is applied in four steps. First, no-data pixels are corrected. Then MNF transformation is used to spatially decorrelate and segregate noise from random noise-dominated bands. Further, random noise from all the bands using local spatial statistical techniques are identified and corrected. Subsequently, local quadratic regression by a least squares method-based technique is used to detect and correct global vertical stripes. Finally, local brightness levels normalization from nearest neighbors using local spatial statistics are used to detect and correct local vertical stripes. The proposed method has been tested on two Hyperion images from Udaipur, western India, and Luleå, northern Sweden. The results and outcomes show that the proposed method efficiently removes noise, primarily from the bands inside or close to the water vapor absorption region, which are very useful and predominantly significant for geological applications.

\section{References}

1. F. D. Van der Meer et al., "Multi-and hyperspectral geologic remote sensing: a review," Int. J. Appl. Earth Obs. Geoinf. 14(1), 112-128 (2012).

2. T. Han et al., "Detection and correction of abnormal pixels in Hyperion images," in IEEE 2002 Int. Geosci. and Remote Sens. Symp., IGARSS 2002, Toronto, Canada, pp. 1327-1330 (2002).

3. D. White, Hyperion Tools 2.0 Installation and User Guide, TRW Space, Defense \& Information Systems, Redondo Beach, California (2011).

4. S. Adler-Golden et al., "Spectral image destriping using a low-dimensional model," Proc. SPIE 8743, 87431Q (2013).

5. H. Li et al., "Research of image preprocessing methods for EO-1 Hyperion hyperspectral data in tidal flat area," Proc. SPIE 7147, 71471G (2008).

6. D. Scheffler and P. Karrasch, "Destriping of hyperspectral image data: an evaluation of different algorithms using EO-1 Hyperion data," J. Appl. Remote Sens. 8(1), 083645 (2014).

7. D. G. Goodenough et al., "Processing Hyperion and ALI for forest classification," IEEE Trans. Geosci. Remote Sens. 41(6), 1321-1331 (2003).

8. B. Datt et al., "Preprocessing EO-1 Hyperion hyperspectral data to support the application of agricultural indexes," IEEE Trans. Geosci. Remote Sens. 41(6), 1246-1259 (2003).

9. H. Sheng and L. Zhang, "A MAP-based algorithm for destriping and inpainting of remotely sensed images," IEEE Trans. Geosci. Remote Sens. 47(5), 1492-1502 (2009). 
Pal, Porwal, and Rasmussen: Noise reduction and destriping using local spatial statistics...

10. M. M. Bouali, "Destriping data from multidetector imaging spectrometers: a study on the MODIS instrument," PhD thesis, TELECOM ParisTech (2011).

11. A. A. Green et al., "A transformation for ordering multispectral data in terms of image quality with implications for noise removal," IEEE Trans. Geosci. Remote Sens. 26, 65-74 (1988).

12. C. Chang and Q. Du, "Interference and noise-adjusted principal components analysis," IEEE Trans. Geosci. Remote Sens. 37(5), 2387-2396 (1999).

13. Q. Yuan, L. Zhang, and H. Shen, "Hyperspectral image denoising employing a spectralspatial adaptive total variation model," IEEE Trans. Geosci. Remote Sens. 50(10), 3660-3677 (2012).

14. L. Gao et al., "A comparative study on linear regression-based noise estimation for hyperspectral imagery," IEEE J. Sel. Top. Appl. Earth Obs. Remote Sens. 6(2), 488-498 (2013).

15. X. Guo et al., "Hyperspectral image noise reduction based on rank-1 tensor decomposition," ISPRS J. Photogramm. Remote Sens. 83, 50-63 (2013).

16. Y. Yuan, X. Zheng, and X. Lu, "Spectral-spatial kernel regularized for hyperspectral image denoising," IEEE Trans. Geosci. Remote Sens. 53(7), 3815-3832 (2015).

17. D. Schläpfer and R. Richter, "Spectral polishing of high-resolution imaging spectroscopy data," presented at 7th SIG-IS Workshop on Imaging Spectrosc., Edinburgh, p. 7 (2011).

18. R. A. Leathers and T. V. Downes, "Scene-based nonuniformity correction and bad-pixel identification for hyperspectral VNIR/SWIR sensors," in IGARSS, pp. 2373-2376 (2006).

19. A. D. Fischer, T. V. Downes, and R. Leathers, "Median spectral-spatial bad pixel identification and replacement for hyperspectral SWIR sensors," Proc. SPIE 6565, 65651E (2007).

20. B. Aiazzi, L. Alparone, and S. Baronti, "Signal-dependent noise modeling for adaptive multiresolution local-statistics filtering," Proc. SPIE 3646, 207-216 (1999).

21. B. Aiazzi et al., "Benefits of signal-dependent noise reduction for spectral analysis of data from advanced imaging spectrometers," in Third Workshop Hyperspectral Image and Signal Process.: Evol. in Remote Sens., IEEE, pp. 1-4 (2011).

22. J. Chen et al., "Oblique striping removal in remote sensing imagery based on wavelet transform,” Int. J. Remote Sens. 27(8), 1717-1723 (2006).

23. J. G. Liu and G. L. K. Morgan, "FFT selective and adaptive filtering for removal of systematic noise in ETM+ imageodesy images," IEEE Trans. Geosci. Remote Sens. 44(12), 3716-3724 (2006).

24. R. Srinivasan, M. Cannon, and J. White, "Landsat data destriping using power spectral filtering," Opt. Eng. 27(11), 271193 (1988).

25. B. Munch et al., "Stripe and ring artifact removal with combined wavelet-Fourier filtering," Opt. Express 17(10), 8567-8591 (2009).

26. R. Pande-Chhetri and A. Abd-Elrahman, "De-striping hyperspectral imagery using wavelet transform and adaptive frequency domain filtering," ISPRS J. Photogramm. Remote Sens. 66(5), 620-636 (2011).

27. M. Cannon, A. Lehar, and F. Preston, "Background pattern removal by power spectral filtering," Appl. Opt. 22(6), 777-779 (1983).

28. R. Pande-Chhetri and A. Abd-Elrahman, "Filtering high-resolution hyperspectral imagery in a maximum noise fraction transform domain using wavelet-based de-striping," Int. J. Remote Sens. 34(6), 2216-2235 (2013).

29. F. A. Kruse, J. Boardman, and J. Huntington, "Comparison of airborne hyperspectral data and EO-1 Hyperion for mineral mapping," IEEE Trans. Geosci. Remote Sens. 41(6), 13881400 (2003).

30. F. A. Kruse, "Use of airborne imaging spectrometer data to map minerals associated with hydrothermally altered rocks in the Northern Grapevine Mountains, Nevada, and California," Remote Sens. Environ. 24, 31-51 (1988).

31. B. K. P. Horn and R. J. Woodham, "Destriping Landsat MSS images using histogram modification," Comput. Graphics Image Process. 10(1), 69-83 (1979).

32. X. Lu, Y. Wang, and Y. Yuan, "Graph-regularized low-rank representation for destriping of hyperspectral images,” IEEE Trans. Geosci. Remote Sens. 51(7), 4009-4018 (2013). 
Pal, Porwal, and Rasmussen: Noise reduction and destriping using local spatial statistics...

33. Y. S. Xie, J. N. Wang, and K. Shang, "An improved approach based on moment matching to destriping for Hyperion data," Proc. Environ. Sci. 10, 319-324 (2011).

34. N. Acito, M. Diani, and G. Corsini, "Subspace-based striping noise reduction in hyperspectral images," IEEE Trans. Geosci. Remote Sens. 49(4), 1325-1342 (2011).

35. J. D. Dykstra and D. B. Segal, "Analysis of AIS data of the Recluse Oil Field, Recluse, Wyoming," in Proc. AIS Workshop, JPL Publication 85-41, Jet Propulsion Laboratory, Pasadena, California, pp. 86-91 (1985).

36. M. Wegener, "Destriping multiple sensor imagery by improved histogram matching," Int. J. Remote Sens. 11(5), 859-875 (1990).

37. B. Zhu et al., "Review on methods for SNR estimation of optical remote sensing imagery," Remote Sens. Technol. Appl. 25(2), 303-309 (2010).

38. G. Lidong and L. Guoqing, "Research on method of remote sensing data quality contrast among different quantization levels," in Int. Conf. Remote Sens. Environ. and Transp. Eng., Atlantis Press (2013).

39. J. Chen et al., "Destriping CMODIS data by power filtering," IEEE Trans. Geosci. Remote Sens. 41(9), 2119-2124 (2003).

40. P. Rakwatin, W. Takeuchi, and Y. Yasuoka, "Stripe noise reduction in MODIS data by combining histogram matching with facet filter," IEEE Trans. Geosci. Remote Sens. 45(6), 1844-1856 (2007).

41. J. J. Simpson, J. I. Gobat, and R. Frouin, "Improved destriping of GOES images using finite impulse response filters," Remote Sens. Environ. 52(1), 15-35 (1995).

42. G. G. Corsini, M. M. Diani, and T. Walzel, "Striping removal in MOS-B data," IEEE Trans. Geosci. Remote Sens. 38, 1439-1446 (2000)

43. B. Zhu et al., "A new method based on spatial dimension correlation and fast Fourier transform for SNR estimation in remote sensing images," in IEEE Int. Geosci. and Remote Sens. Symp. (IGARSS), IEEE, pp. 4114-4117 (2013).

44. Z. P. Sun et al., "Image quality evaluation of HJ-1 satellite CCD sensor," Infrared 31(9), 30-36 (2010).

Biographies of the authors are not available. 